\title{
Investigation of the light nuclei spin structure from hadronic channels at NUCLOTRON
}

\author{
P.K.Kurilkin*1, I.Dobrin ${ }^{2}$, V.V.Fimushkin ${ }^{1}$, D.A.Finogenov ${ }^{3}$, S.G.Genchev ${ }^{4}$, \\ Yu.V.Gurchin ${ }^{1}$, A.P.lerusalimov ${ }^{1}$, A.Yu.Isupov ${ }^{1}$, K.Itoh ${ }^{5}$, M.Janek ${ }^{6}$, E.V.Karpechev ${ }^{3}$, \\ J.-T.Karachuk ${ }^{1,2}$, S.V.Khabarov ${ }^{1}$, T.Kawabata ${ }^{7}$, A.N.Khrenov ${ }^{1}$, V.A.Krasnov ${ }^{1}$,
} A.B.Kurepin $^{3}$, A.K.Kurilkin ${ }^{1}$, V.P.Ladygin ${ }^{1}$, N.B.Ladygina ${ }^{1}$, D.Lipchinski ${ }^{2}$, A.N.Livanov ${ }^{1}$, Y.Maeda ${ }^{8}$, A.I.Malakhov ${ }^{1}$, G.Martinska ${ }^{9}$, S.M.Piyadin ${ }^{1}$, J.Popovichi ${ }^{2}$, A.N.Prokofichev ${ }^{1}$, V.L.Rapatsky ${ }^{1}$, A.I.Reshetin ${ }^{3}$, S.G.Reznikov ${ }^{1}$, P.A.Rukoyatkin ${ }^{1}$, S.Sakaguchi ${ }^{10}$, H.Sakai ${ }^{11,12}$, Y.Sasamoto ${ }^{13}$, K.Sekiguchi ${ }^{14}$, Ya.G.Skhomenko ${ }^{15}$, K.Suda ${ }^{12}$, V.V.Syschenko ${ }^{15}$, G.Tarjányiová ${ }^{6}$, A.A.Terekhin ${ }^{1}$, T.Uesaka ${ }^{12}$, J.Urban ${ }^{9}$, T.A.Vasiliev ${ }^{1}$, I.E.Vnukov ${ }^{15}$, N.I.Zamiatin ${ }^{1}$, E.V.Zubarev ${ }^{1}$

(DSS collaboration)

${ }^{1}$ Joint Institute for Nuclear Research, Dubna 141980, Russian Federation

${ }^{2}$ Advanced Research Institute for Electrical Engineering, Bucharest 030138, Romania

${ }^{3}$ Institute for Nuclear Research of RAS, Moscow 117312, Russian Federation

${ }^{4}$ Laboratory for Technical Developments and Applications, BAS, Sofia 1784, Bulgaria

${ }^{5}$ Department of Physics, Saitama University, Saitama 338-8570, Japan

${ }^{6}$ Physics Department, University of Žilina, Žilina 010 26, Slovak Republic

${ }^{7}$ Department of Physics, Kyoto University, Kyoto 606-8502, Japan

${ }^{8}$ Department of Applied Physics, University of Miyazaki, Miyazaki 889-2192, Japan

${ }^{9}$ P.J.Šafarik University, Košice 041 80, Slovak Republic

${ }^{10}$ Department of Physics, Kyushu University, Fukuoka 812-8581, Japan

${ }^{11}$ Department of Physics, University of Tokyo, Tokyo 113-8654, Japan

${ }^{12}$ RIKEN Nishina Center, Saitama 351-0198, Japan

${ }^{13}$ Center for Nuclear Study, University of Tokyo, Tokyo 113-0033, Japan

${ }^{14}$ Department of Physics, Tohoku University, Sendai 980-8578, Japan

${ }^{15}$ Belgorog National Research University, Belgorod 308015, Russian Federation

E-mail: pkurilkinejinr.ru

\begin{abstract}
The wide experimental program of the DSS project devoted to the investigation of the spin structure of light nuclei using internal and extracted deuteron beam at Nuclotron(JINR, Dubna) is discussed. Recent data on the deuteron analyzing powers in $d p$ - elastic scattering obtained at Nuclotron demonstrate strong deviation from the relativistic multiple scattering model predictions at large scattering angle in the cms. The scheme of the deuteron beam polarimetry at Nuclotron at intermediate and low energies is discussed.
\end{abstract}

XXII International Baldin Seminar on High Energy Physics Problems,

15-20 September 2014

JINR, Dubna, Russia

* Speaker. 


\section{Introduction}

The data of light nuclei interaction in the reactions induced by unpolarized and polarized deuterons are very important to describe the nature of $2 N$ and $3 N$ correlation, the relativistic effects and nonnucleon degrees of freedom. During last several decades the investigation of the cross section and polarization observables in $N d$ interaction have been performed at different experiments all over the world at RIKEN[1]-[4], KVI[5]-[11], IUCF[12]-[14] and RCNP[15, 16] at intermediate $(E / A \sim 100-300 \mathrm{MeV})$ energies.

The cross section and polarization observables data in $N d$ - elastic scattering have been accumulated at the energy range $130-270 \mathrm{MeV}$ [1]-[14]. Such activities were stimulated by the observed discrepancy of $30 \%$ between the differential cross section data[1, 17] and Faddeev calculation results[18] using modern high-quality nucleon-nucleon (NN) potentials CD-Bonn [19], Argone-V18 (AV18) [20], Nijmegen-I and Nijmegen-II[21]. It was found that the discrepancies between the Faddeev calculations and experimental data on the cross section and polarization observables increase with the energy at large scattering angles. The inclusion of the $2 \pi$ - exchange three-nucleon forces (3NF) such as TM-3NF [22], TM99[23] or UrbanaIX -3NF [24] does not help remove these discrepancies at the energies higher than $250 \mathrm{MeV}[15,16,25,26]$ at backward angles around $\Theta_{c m} \sim 140^{\circ}$. The authors stated that the reason of this discrepancy can be neglecting by new type of short-range $3 \mathrm{NF}$. The relativistic effects can also play a role at these energies. The inclusion of the relativistic effects into the calculation leads to improve of the description of experimental data only at very backward scattering angles [27, 28]. So the further investigations of light nuclei interaction processes at higher energies are very desirable to get additional information about internal structure of a nucleon and relativistic effects.

The experimental program of DSS project[29]-[32] includes several parts. First one is to obtain the information on the spin-dependent parts of 2-nucleon and 3-nucleon forces from two processes: $d p$-elastic scattering in a wide energy range from $300 \mathrm{MeV}$ up to $2000 \mathrm{MeV}$ and $d p$ non-mesonic breakup with two protons detection at energies $200-500 \mathrm{MeV}$. The second goal is the measurement of the $T_{20}$ and $C_{y y}$ for the ${ }^{3} \mathrm{He}(d, p)^{4} \mathrm{He}$ reaction[33]-[35] at the energy range of 1000-2000 MeV using extracted polarized deuteron beam from new polarized ion source [36] and polarized ${ }^{3} \mathrm{He}$ target[37]. The development of the high precision deuteron beam polarimetry at low and moderate high energies is important for these investigations.

\section{Recent result obtained at Nuclotron}

The experimental program on the deuteron structure investigation at Nuclotron was started by the measurements of the vector $A_{y}$ and tensor $A_{y y}$ and $A_{x x}$ analyzing powers in $d p$ - elastic scattering at $T_{d}$ of $880 \mathrm{MeV}$ [38, 39] and $2000 \mathrm{MeV}$ [40]. The details of the experiment can be found in [41]. The detection system (see Fig.1) was designed for analyzing powers measurements in a wide range of initial deuteron energies. The detector support with mounted 46 plastic scintillation counters was placed downstream the ITS spherical chamber. Each plastic scintillation counter was coupled to a photo-multiplier tube Hamamatsu H7416MOD. Nine proton detectors were installed for left, right and up, but due to space limitation - only four for down directions. Four deuteron detectors were placed at scattering angles of deuterons coinciding kinematically with the protons for left, right and 


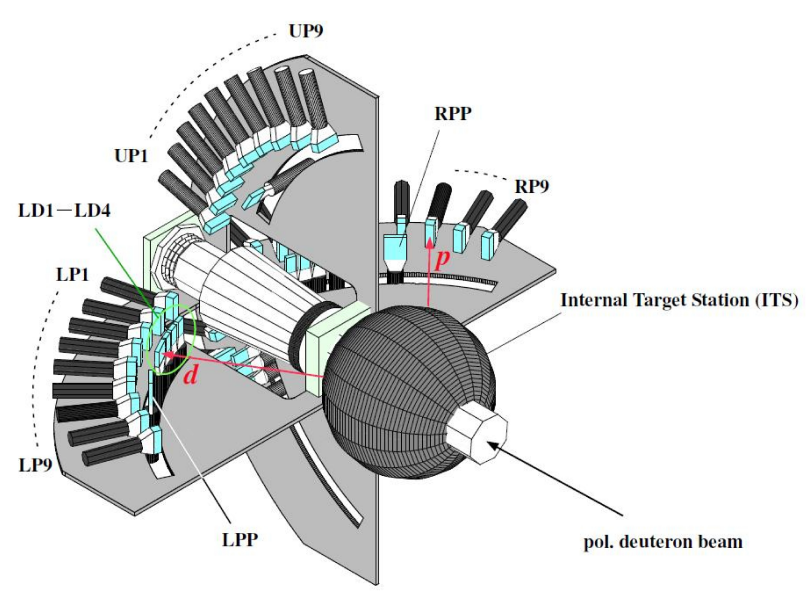

Figure 1: A schematic view of the experimental setup installed downstream the ITS spherical chamber for analyzing powers measurements. Plastic scintillation counters coupled to PMTs are placed to the left, right, up, and down the beam axis.

up scattering. Only one deuteron detector was used to cover the solid angle corresponding to down scattering. In addition, one pair of detectors was placed to register two protons from quasi-elastic $p p$ - scattering at $\theta_{p p}=90^{\circ}$ in the c.m. in the horizontal plane to monitor the beam luminosity. Selection of the $d p$ - elastic scattering events was based on the kinematical coincidence of the scattered deuterons and recoil protons by the scintillation counters. The energy loss correlation and time-of-flight difference for the signals from the corresponding proton-deuteron detector pairs were used in the analysis.

The beam polarization has been measured before the data taking using the asymmetry of the $d p$ - elastic scattering yields at $T_{d}^{l a b}=270 \mathrm{MeV}$ with the same detection system [42] and the high precision data on analyzing powers for the reaction obtained at RIKEN[3, 43].

The angular dependencies of the vector $A_{y}$ and tensor analyzing powers $A_{y y}$ and $A_{x x}$ obtained at $T_{d}^{l a b}=880 \mathrm{MeV}$ are presented in Fig. 2 by the solid symbols. The error bars are the statistical only. The solid lines in Fig. 2 are the results of the nonrelativistic Faddeev calculations[44] using the CDBonn nucleon-nucleon potential[19]. The dashed lines correspond to a relativistic calculation in the multiple scattering expansion formalism [45] up to the second-order terms of the nucleon-nucleon $t$-matrix [46] with the use of the CD-Bonn [19] DWF. The model [45] takes into account the offenergy-shell effects. The dot-dashed curves correspond to the relativistic calculation of the optical potential framework [47] up to the total angular momentum $J=39 / 2$. The results are obtained with the use of DWF derived from the dressed bag model of the Moscow-Tübingen group [48] and the on-shell nucleon-nucleon $t$-matrix based on the phase-shift analysis solution SP07 [49]. One can see that the results of Faddeev calculations based on CD-Bonn potential even without involving $3 \mathrm{NFs}$ are in good agreement with all the analyzing powers within the achieved experimental accuracy while the approach [45] describes reasonably well the angular dependencies of $A_{y}$ over the whole angular range of measurements and $A_{y y}$ at backward angles only. The calculations [47] reproduce only the behavior of tensor analyzing powers $A_{y y}$ at the angles larger than $100^{\circ}$ in the c.m., while they fail to describe the analyzing powers $A_{y}$ and $A_{x x}$. It can be noted, that the relativis- 

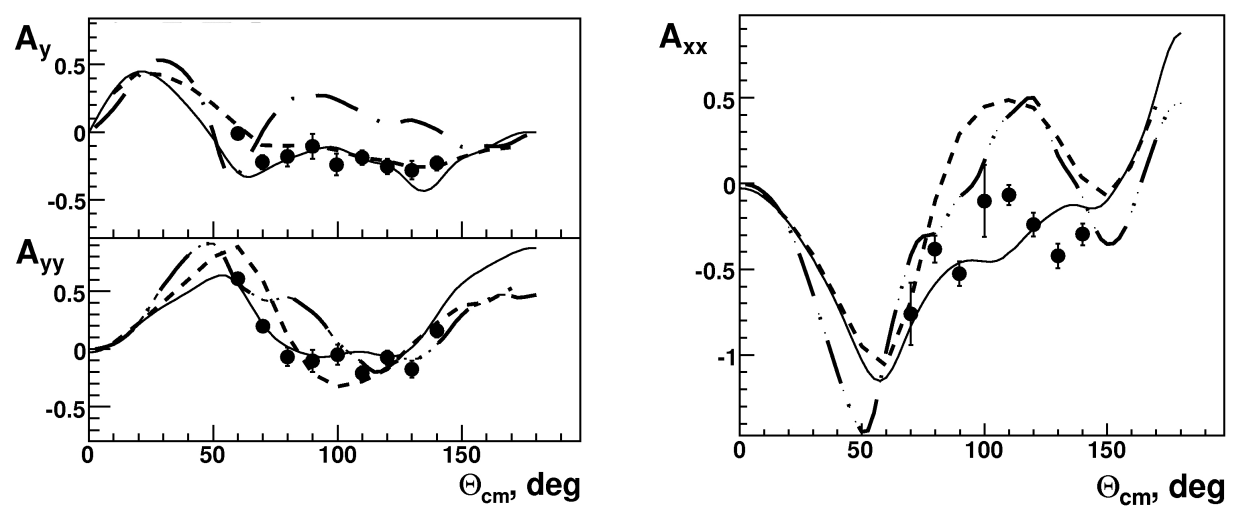

Figure 2: The angular dependencies of the vector $A_{y}$ and tensor analyzing powers $A_{y y}$ and $A_{x x}$ for $d p$ - elastic scattering at $T_{d}^{l a b}=880 \mathrm{MeV}$. The lines are explained in the text.

tic multiple scattering model calculations $[45,50]$ give the better agreement with the differential cross section data in the vicinity of its minimum than the nonrelativistic Faddeev calculations at this energy[39].

The results on the angular dependence of the vector $A_{y}$ and tensor $A_{y y}$ analyzing powers in $d p-$ elastic scattering at $2000 \mathrm{MeV}$ are shown in Figures 3 and 4, respectively. The data obtained at Argonne National Laboratory(ANL) are presented by the solid symbols[51]. Open squares and circles are the data obtained at the ITS[40] and at hydrogen bubble chamber at JINR[54], respectively. The dashed and solid lines are the results of the relativistic multiple scattering model calculations[45] with and without of the double scattering term. The full calculations are in a reasonable agreement with the data.

The dependence of the vector $A_{y}$ and tensor $A_{y y}$ analyzing powers at fixed angles in c.m.s are plotted as function of transverse momentum $p_{T}$ in the Fig.5 and Fig.6, respectively. The open and solid symbols represent the data obtained at RIKEN [1, 3, 4], Saclay [55, 56], ANL [51]-[53] and at Nuclotron [39, 40], respectively. The values of $A_{y y}$ are change the sign at $p_{T} \sim 600-650 \mathrm{MeV} / \mathrm{c}$ as in the case of deuteron inclusive breakup. The negative sign of $A_{y y}$ is observed at large $p_{T}$. Vector

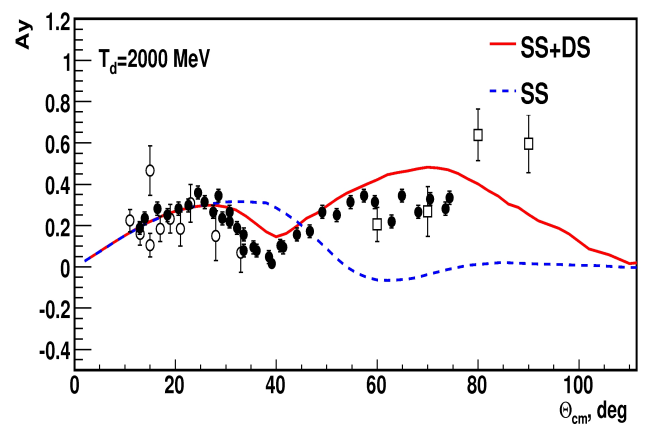

Figure 3: Vector $A_{y}$ analyzing power in $d p$ elastic scattering at $2000 \mathrm{MeV}$. The symbols and curves are explained in the text.

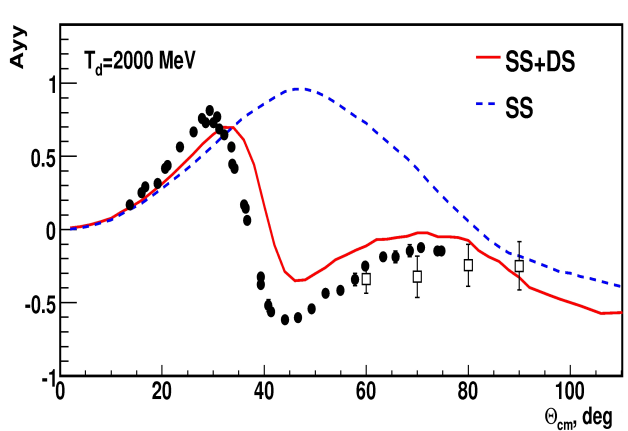

Figure 4: Tensor $A_{y y}$ analyzing power in $d p$ elastic scattering at $2000 \mathrm{MeV}$. The symbols and curves are the same as in Figure 3 


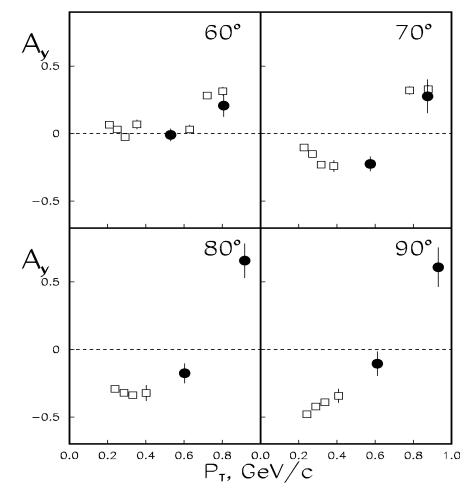

Figure 5: Vector $A_{y}$ analyzing power in $d p-$ elastic scattering obtained at fixed angles of $60^{\circ}$, $70^{\circ}, 80^{\circ}$ and $90^{\circ}$ in the c.m. as a function of transverse momentum $p_{T}$. The open and solid symbols are the data obtained at RIKEN [1, 3, 4], Saclay [55, 56], ANL [51]-[53] and at Nuclotron [39, 40], respectively.

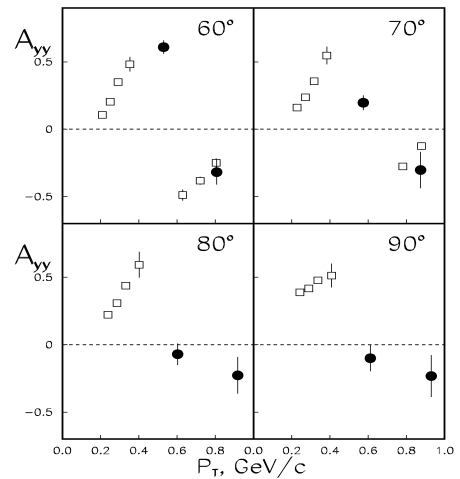

Figure 6: Tensor $A_{y} y$ analyzing power in $d p-$ elastic scattering obtained at fixed angles of $60^{\circ}$, $70^{\circ}, 80^{\circ}$ and $90^{\circ}$ in the c.m. as a function of transverse momentum $p_{T}$. The open and solid symbols are the data obtained at RIKEN [1, 3, 4], Saclay [55, 56], ANL [51]-[53] and at Nuclotron $[39,40]$, respectively.

analyzing power $A_{y}$ has small negative values at low $p_{T}$, but it achieves large positive values at $p_{T}$ higher $\sim 700 \mathrm{MeV} / \mathrm{c}$. The change of the sign is also observed for $A_{y}$ at $p_{T} \sim 600-700 \mathrm{MeV} / \mathrm{c}$ at large scattering angles in the c.m. The large positive values of the single spin asymmetry is also observed in $p p$ - elastic scattering at high energies and large $p_{T}$ (so called Krish-effect[57]). It would be interesting to perform the further precise measurement to understand the reason of such behavior.

\section{Deuteron beam polarimetry at Nuclotron}

The main part of experimental program of DSS project is related with the use of polarized deuteron beam. For these investigations the high precision deuteron beam polarimetry at Nuclotron accelerator complex is necessary to obtain reliable values of beam polarization.

The polarimeter based on the spin asymmetry measurement in $d p$ - elastic scattering at large angles $\left(\theta_{\mathrm{cm}} \geq 60^{\circ}\right)$ at $270 \mathrm{MeV}$ [42], where precise data on analyzing powers [3, 4] exist, and placed at internal target station (ITS) at Nuclotron is proposed as the reference deuteron polarimeter at Nuclotron-NICA. The accuracy of the determination of the deuteron beam polarization achieved with this method is better than $2 \%$ because of the values of the analyzing powers were obtained for the polarized deuteron beam, which absolute polarization had been calibrated via the ${ }^{12} \mathrm{C}(d, \alpha){ }^{10} \mathrm{~B}^{*}\left[2^{+}\right]$reaction [43]. This polarimeter is planned to use in the counting mode for permanent monitoring of the beam polarization at the energies of $270-2000 \mathrm{MeV}[41,58]$.

We propose to design new low energy deuteron polarimeter based on the detection of the charged particles from the $d d \rightarrow^{3} H p$ reaction at $10 \mathrm{MeV}$. Both deuteron vector and tensor analyzing powers at the scattering angle of $\sim 130^{\circ}$ in the c.m. [59, 60] are large enough to provide the efficient polarimetry. The thin solid $C D_{2}$ and carbon targets will be used to obtain the effect 


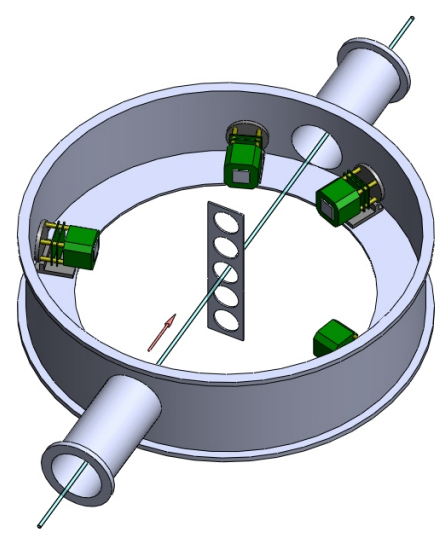

Figure 7: A schematic view of the deuteron beam polarimeter at $10 \mathrm{MeV}$.

on deuterium via $C D_{2}-C$ subtraction procedure. This will avoid the use of the gaseous targets. However, the problem of the carbon background elimination appears.

The scheme of the low energy deuteron polarimeter with solid target and 4 silicon strip detectors for the detection of the scattering on the left and right is shown in Fig.7.

The proton and triton will be detected by the two silicon microstrip detectors developed at LHEP JINR [61]. The strip size is $500 \mu \mathrm{m}$ with the number of strips 64 in the both $\mathrm{X}$ and $\mathrm{Y}$ directions. The selection events of the $d d \rightarrow p^{3} \mathrm{H}$ reaction will be done using the proton and triton scattering angles correlation and complanarity condition. These selection criteria will significantly reduce the the contribution from the carbon content of the $\mathrm{CD}_{2}$ target. The additional selection can be the energy depositions of the proton and triton in the silicon detector. The simulation of the $d d \rightarrow p^{3} \mathrm{H}$ events selection using the relation between scattering angles of protons and tritons and at $130^{\circ}$ in the c.m. at $10 \mathrm{MeV}$ and distance of $20 \mathrm{~cm}$ from the target has been performed using Pluto event generator and ROOT package. The background coming from $d d \rightarrow d d, d d \rightarrow$ pnpn and $d d \rightarrow d p n$ channels has been taken into account. The correlations of the $\mathrm{X}$ and $\mathrm{Y}$ coordinates for the proton and triton detectors are presented in Figs. 8 and 9, respectively. One can see the clean selection of the $d d \rightarrow{ }^{3} \mathrm{H} p$ events. The energy loss information will be also used in the analysis to reduce the background.

\section{Future plans of DSS experiment at Nuclotron}

The first stage of the DSS experimental program[30, 31, 32] is the beam energy scan of $d p$ elastic scattering cross section at the deuteron energies from 400 up to $2000 \mathrm{MeV}$ and measurements of $d p$ - non-mesonic breakup at 300,400 and $500 \mathrm{MeV}$ in different kinematic configurations. These measurements were performed using ITS[62] with new control and data acquisition system[63]. The details and preliminary results of the experiments on the study of the energy dependence of $d p$ - elastic scattering cross section and investigation of $d p \rightarrow p p n$ reaction can be found in $[64,65]$ and $[67,68]$, respectively. 


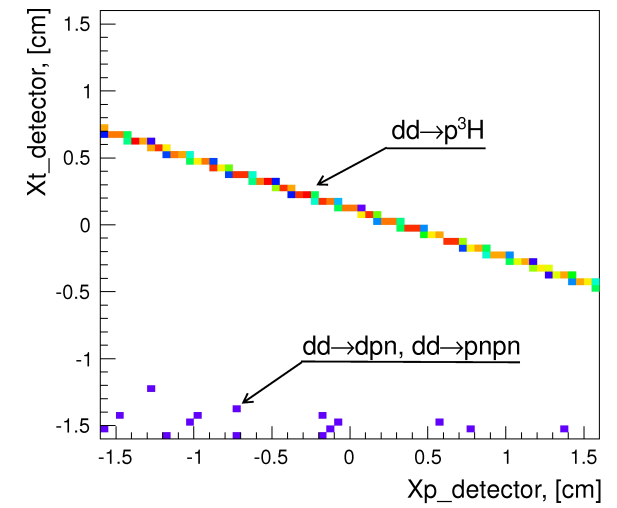

Figure 8: The correlation of the $\mathrm{X}$-coordinates for protons and tritons registered by the silicon strip detector.

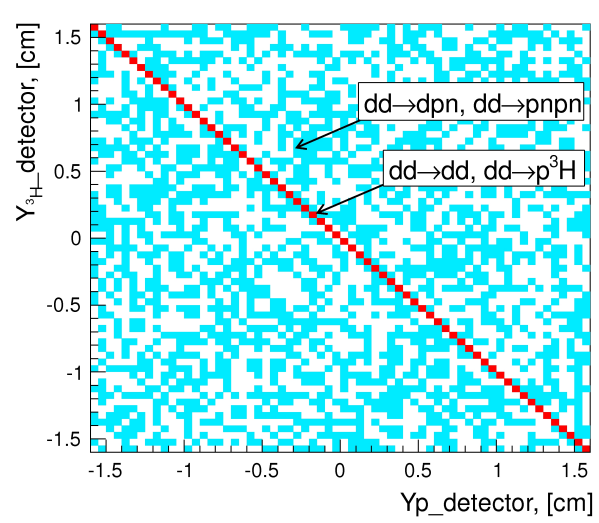

Figure 9: The correlation of the Y-coordinates for protons and tritons registered by the silicon strip detector.

Future plans of DSS collaborations in spin studies are related with the construction of new polarized deuteron source[36]. In our experiments we are planning to use the spin modes with the following ideal values of $\left(p_{z}, p_{z z}\right):(0,0),(0,-2),(2 / 3,0)$ and $(-1 / 3,+1)$.

The analyzing powers measurements in $d p$ - elastic scattering at large angles in $\mathrm{cms}$ at various energies up to $2000 \mathrm{MeV}$ and in $d p$-non-mesonic breakup at the energies below $500 \mathrm{MeV}$ will be done using internal target and polarized deuteron beam from new PIS.

The main goal of the DSS experiment is to obtain the data on $C_{/ /}$in the energy region of $1.0-1.75 \mathrm{GeV}$, where the contribution from the deuteron $\mathrm{D}$-state is expected to reach a maximum in one-nucleon exchange approximation. It is necessary to obtain new information on the strange structure observed in the behaviour of $T_{20}$ in the $d p$ - backward elastic scattering and to realize experiment on the full determination of the matrix element of the ${ }^{3} \mathrm{He}(d, p){ }^{4} \mathrm{He}$ reaction in the model independent way. These data will help us also to understand the short-range spin structure of deuteron and effects of non-nucleonic degrees of freedom. For these purposes the extracted polarized deuteron beam from new PIS[36] and spin-exchange-type polarized ${ }^{3} \mathrm{He}$ target developed at CNS of Tokyo University[37] and modified for the experiment at Nuclotron can be used.

For these investigation it is planned to perform the calibration of the ITS polarimeter at the energies 270-2000 MeV [41, 69, 70, 71] with the expected error bars for analyzing powers \pm 0.02 and the calibration of the high energy polarimeters based on the $d p$ - elastic scattering at forward angles where both tensor and vector analyzing powers have large values $[52,56]$. The possibility of the $d p$ - elastic scattering events selection using timing and amplitude information from the scintillation counters detected deuterons and protons in the kinematic coincidence has been demonstrated at $1600 \mathrm{MeV}$ and $2000 \mathrm{MeV}$ [72].

\section{Conclusion}

The data on the analyzing powers $A_{y}, A_{y y}$ and $A_{x x}$ in $d p$ - elastic scattering have been measured at ITS at the Nuclotron at the energies of 880 and $2000 \mathrm{MeV}$. The results on the tensor analyzing powers at $880 \mathrm{MeV}$ indicate strong deviations from the predictions of the relativistic 
phenomenological approaches $[45,47]$ based on the use of the nucleon-nucleon forces only. The non-relativistic Faddeev calculations [44] using CD-Bonn 2NF [19] describe only the angular behavior of the analyzing powers data, however, they fail to reproduce the differential cross section data obtained in earlier experiments [73, 74].

Future studies of the deuteron-induced reactions like $d p \rightarrow p d, d p$ - breakup, $d d \rightarrow{ }^{3} \mathrm{Hp}\left({ }^{3} \mathrm{Hen}\right)$ and $d^{3} \mathrm{He} \rightarrow p^{4} \mathrm{He}$ at Nuclotron are related with new PIS developed at LHEP-JINR. For these investigations it is necessary to develop the scheme of the deuteron beam polarization measurements. The polarimeter at ITS [42] should play a important role being the reference deuteron polarimeter at Nuclotron. It will provide the precision in the deuteron beam polarization $\sim 3 \%$ at the energy range of 270-2000 MeV. The new low energy deuteron beam polarimeter based on the detection of the charged particles from the $d d \rightarrow^{3} \mathrm{Hp}$ reaction at $10 \mathrm{MeV}$ is proposed to measurement of the tensor and vector polarization component of the deuteron beam simultaneously.

\section{Acknowledgments}

The work has been supported in part by the programs of the scientific cooperation between JINR and Slovak Republik in 2014y. and by the RFBR grant No.13-02-00101a.

\section{References}

[1] N. Sakamoto et al., Measurement of the vector and tensor analyzing powers for the d-p elastic scattering at $E_{d}=270 \mathrm{MeV}$, Phys.Lett. $\mathbf{B 3 6 7}$ (1996) 60.

[2] H. Sakai et al., Precise Measurement of deuteron proton Elastic Scattering at 270-MeV and Three Nucleon Force Effects, Phys.Rev.Lett. 84 (2000) 5288.

[3] K. Sekiguchi et al., Complete set of precise deuteron analyzing powers at intermediate energies: Comparison with modern nuclear force predictions, Phys.Rev. C65(2002) 034003.

[4] K. Sekiguchi et al., Polarization transfer measurement for ${ }^{1} H(\vec{d}, \vec{p})^{2} H$ elastic scattering at 135-MeV/u and three nucleon force effects, Phys.Rev. C70 (2004) 014001.

[5] R. Bieber et al., Three-Nucleon Force and the Ay Puzzle in Intermediate Energy $\vec{p}+d$ and $\vec{d}+p$ Elastic Scattering, Phys. Rev. Lett. 84 (2000) 606.

[6] K. Ermisch et al., Search for Three-Nucleon Force Effects in Analyzing Powers for $\vec{p} d$ Elastic Scattering, Phys. Rev. Lett. 86 (2001) 5862.

[7] K. Ermisch et al., Systematic investigation of three-nucleon force effects in elastic scattering of polarized protons from deuterons at intermediate energies, Phys. Rev. C71 (2005) 064004.

[8] E. Stephan et al., Vector and tensor analyzing powers of elastic deuteron-proton scattering at 130-MeV deuteron beam energy, Phys.Rev. C76 (2007) 057001.

[9] H. Mardanpour et al., Precision measurement of vector and tensor analyzing powers in elastic deuteron-proton scattering, Eur.Phys.J. A31 (2007) 383.

[10] H.R. Amir-Ahmadi et al., Three-nucleon force effects in cross section and spin observables of elastic deuteron-proton scattering at 90 MeV/nucleon, Phys.Rev. C75 (2007) 041001(R).

[11] A. Ramazani-Moghaddam-Arani et al., Elastic proton-deuteron scattering at intermediate energies, Phys.Rev. C78 (2008) 014006. 
[12] E.J. Stephenson et al., Indications of three nucleon force effects in the proton analyzing power for 70-MeV to 200-MeV polarized d elastic scattering, Phys.Rev. C60 (1999) 061001.

[13] R.V. Cadman et al., Evidence for a three nucleon force effect in proton - deuteron elastic scattering, Phys.Rev.Lett. 86 (2001) 967.

[14] B.V. Przewoski et al., Analyzing powers and spin correlation coefficients for $p+d$ elastic scattering at 135-MeV and 200-MeV, Phys.Rev. C74 (2006) 064003.

[15] K. Hatanaka et al., Cross-section and complete set of proton spin observables in $\vec{p} d$ elastic scattering at 250-MeV, Phys.Rev. C66 (2002) 044002.

[16] Y. Maeda et al., Differential cross section and analyzing power measurements for $\vec{n} d$ elastic scattering at $248 \mathrm{MeV}$, Phys.Rev. C76 (2007) 014004.

[17] H. Shimizu et al., Analyzing powers and cross section in elastic $\vec{p}-d$ scattering at $65 \mathrm{MeV}$, Nucl.Phys. A382 (1982) 242.

[18] W. Glöckle, H. Witala, D. Huber, H. Kamada, J. Golak, The three-nucleon continuum: achievements, challenges and applications, Phys.Rep. 274 (1996) 107.

[19] R. Machleidt, The high-precision, charge-dependent Bonn nucleon-nucleon potential (CD-Bonn), Phys. Rev. C63 (2001) 024001.

[20] R. B. Wiringa, V. G. J. Stoks, and R. Schiavilla, Accurate nucleon-nucleon potential with charge-independence breaking, Phys. Rev. C51 (1995) 38.

[21] V.G.J. Stoks et al. Construction of high quality NN potential models, Phys. Rev. C49 (1994) 2950.

[22] S.A. Coon, M. Scadron, P. McNamee, B.R. Barrett, D. Blatt, B. McKellar, The Two Pion Exchange, Three Nucleon Potential and Nuclear Matter, Nucl.Phys. A317 (1979) 242.

[23] S.A. Coon, H.K. Han, Reworking the Tucson-Melbourne three nucleon potential, Few-Body Syst. 30 (2001) 131.

[24] B.S. Pudliner, V.R. Pandharipande, J. Carlson, S.C. Pieper and R.B. Wiringa, Quantum Monte Carlo calculations of nuclei with A<=7, Phys.Rev. C56 (1997) 1720.

[25] K. Ermisch et al., Systematic investigation of the elastic proton deuteron differential cross-section at intermediate-energies, Phys. Rev. C68 (2003) 051001.

[26] K. Sekiguchi et al., Three Nucleon Force Effects in Intermediate Energy Deuteron Analyzing Powers for dp Elastic Scattering, Phys. Rev. C83 (2011) 061001.

[27] T. Lin, Ch. Elster, W.N. Polyzou, H. Witala, W. Glöckle, Poincaré Invariant Three-Body Scattering at Intermediate Energies, Phys.Rev. C78 (2008) 024002.

[28] Ch. Elster, T. Lin, W. Glöckle, S. Jeschonnek, Faddeev and Glauber calculations at intermediate energies in a model for $n+d$ scattering, Phys.Rev. C78 (2008) 034002.

[29] V.P. Ladygin et al., Short-range correlations studies in collisions of polarized nuclei at Nuclotron-M, EPJ Web Conferences 3, (2010) 04004.

[30] V.P. Ladygin et al., Recent results with polarized deuterons and polarimetry at Nuclotron-NICA, J.Phys.Conf.Ser. 295 (2011) 012131.

[31] V.P. Ladygin et al., Spin physics in few body systems at Nuclotron, Phys.Part.Nucl. 45 (2014) 327.

[32] V.P. Ladygin et al., Few-body Studies at Nuclotron-JINR, Few Body Syst. 55 (2014) 709. 
[33] T. Uesaka et al., Polarization correlation coefficient for the ${ }^{3} \mathrm{He}(\vec{d}, p){ }^{4} \mathrm{He}$ reaction, Phys.Lett. B467 (1999) 199.

[34] H. Kamada et al., A model for the ${ }^{3} \mathrm{He}(\vec{d}, p){ }^{4} \mathrm{He}$ reaction at intermediate-energies, Prog.Theor.Phys. 104 (2000) 703.

[35] T. Uesaka et al., ${ }^{3} \mathrm{He}(d, p){ }^{4} \mathrm{He}$ reaction at intermediate energies and impulse picture of the $(d, p)$ reactions, Phys. Lett. B533 (2002) 1.

[36] V.V. Fimushkin et al., Source of polarised deuterons, Eur.Phys.J.ST 162 (2008) 275.

[37] T. Uesaka et al., Polarized ${ }^{3}$ He project at RIKEN, Nucl.Instr. and Meth. in Phys.Res. A402 (1998) 212.

[38] P.K.Kurilkin et al., Measurement of the vector and tensor analyzing powers in dp elastic scattering at the energy of $880 \mathrm{MeV}$, Eur.Phys.J. Special Topics 162 (2008) 137.

[39] P.K. Kurilkin et al., Measurement of the vector and tensor analyzing powers for dp-elastic scattering at $880 \mathrm{MeV}$, Phys.Lett. B715 (2012) 61.

[40] P.K. Kurilkin et al., Investigation of the angular dependence of the analyzing powers in the deuteron-proton elastic scattering at the nuclotron, Phys.Part. and Nucl.Lett. 8 (2011) 1081.

[41] T. Uesaka, V.P. Ladygin et al., Proposal on the measurements of d-p elastic scattering analyzing powers at 0.3-2.0 GeV at internal target station of the nuclotron, Part.Nucl. and Nucl. Lett. 3 (2006), 305.

[42] P.K. Kurilkin et al., The $270 \mathrm{MeV}$ deuteron beam polarimeter at the Nuclotron Internal Target Station, Nucl. Instr. Meth. in Phys.Res. A642 (2011) 45.

[43] K. Suda et al., Absolute calibration of the deuteron beam polarization at intermediate energies via the ${ }^{12} C(d$, alpha $){ }^{10} B^{*}[2+]$ reaction, Nucl.Instrum. Methods in Phys.Res. A572 (2007) 745.

[44] H. Witala, W. Glöckle, J. Golak, A. Nogga, H. Kamada, R. Skibinski, J. Kuros-Zolnierczuk, Nd elastic scattering as a tool to probe properties of three nucleon forces, Phys. Rev. C63 (2001) 024007.

[45] N.B. Ladygina, Deuteron-proton elastic scattering at intermediate energies, Phys.Atom.Nucl. 71 (2008) 2039.

[46] N.B. Ladygina, New parameterization of effective nucleon-nucleon t-matrix interaction for scattering at intermediate energies, e-Print: arXiv:0805.3021 [nucl-th].

[47] M.A. Shikhalev, Elastic Nd scattering at intermediate energies as a tool for probing the short-range deuteron structure, Phys.Atom.Nucl. 72 (2009) 588.

[48] V.I. Kukulin, V.N. Pomerantsev, M. Kaskulov, A. Faessler, The properties of the three-nucleon system with the dressed-bag model for NN interaction: I. New scalar three-body force, J.Phys. G: Nucl.Part.Phys. 30 (2004) 287.

[49] http://gwdac.phys.gwu.edu.

[50] N.B. Ladygina, Differential cross section of dp-elastic scattering at intermediate energies, Eur.Phys.J. A42 (2009) 91.

[51] M. Haji-Saied et al., Tensor and vector spin observables in pd elastic scattering at $600 \mathrm{MeV}$, $800 \mathrm{MeV}$, and $1000 \mathrm{MeV}$, Phys.Rev. C36 (1987) 2010.

[52] M.Bleszynski et al., Elastic scattering of 2 GeV polarized deuterons from hydrogen, Phys.Lett. B87 (1979) 198 
[53] M.Bleszynski et al., Evidence for the noneikonal effects in the tensor and vector analyzing powers in $p-\vec{d}$ elastic scattering at $800 \mathrm{MeV}, \mathbf{B 1 0 6}$ Phys.Lett. (1981) 42.

[54] V.V. Glagolev, V.P. Ladygin, N.B. Ladygina and A.A. Terekhin, Measurement of the differential cross-section and deuteron vector analyzing power in dp-elastic scattering at $2.0 \mathrm{GeV}$, Eur.Phys.J. A48 (2012) 182.

[55] M.Garçon et al., Measurements of vector and tensor analyzing powers for 191 and $395 \mathrm{MeV}$ deuteron scattering, Nucl. Phys. A458 (1986) 287.

[56] V. Ghazikhanian et al., Vector and tensor spin observables in the reaction ${ }^{1} \vec{H}(\vec{d}, d)^{1} \vec{H}$ at 1.6-GeV Phys.Rev. C43 (1991) 1532.

[57] A.D.Krisch, Hard collisions of spinning protons: Past, present and future., Eur.Phys.J. A31 (2007) 423.

[58] P. Kurilkin, V.Ladygin. Deuteron beam polarimetry at the nuclotron, Phys.Part.Nucl. 45 (2014) 265.

[59] W. Gruebler et al., Investigation of excited states of ${ }^{4} \mathrm{He}$ via the ${ }^{2} \mathrm{H}(d, p)^{3} \mathrm{H}$ and ${ }^{2} \mathrm{H}(d, n)^{3} \mathrm{He}$ reactions using a polarized deuteron beam, Nucl.Phys. A193 (1972) 129.

[60] V. Konig et al., Investigation of charge symmetry violation in the mirror reactions ${ }^{2} \mathrm{H}(d, p)^{3} \mathrm{H}$ and ${ }^{2} H(d, n)^{3} H e$ Nucl.Phys., $\mathbf{A 3 3 1}$ (1979) 1.

[61] A.V. Isaev et al., First Application of a Double-Side. Multistrip Si-Detector in the GABRIELA Project, PTE 1 (2011) 43.

[62] A.I. Malakhov et al., Potentialities of the internal target station at the Nuclotron, Nucl.Instrum.Meth. in Phys.Res. A440 (2000) 320.

[63] A.Yu. Isupov, V.A. Krasnov, V.P. Ladygin, S.M. Piyadin, S.G. Reznikov, The Nuclotron internal target control and data acquisition system, Nucl.Instrum.Meth. in Phys.Res. A698 (2013) 127.

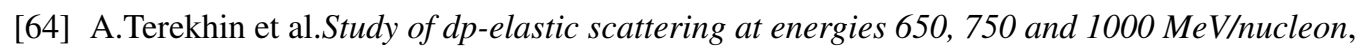
Nucl.Phys.Proc.Suppl. 245 (2013) 185.

[65] Yu.Gurchin et al. The differential cross-section on dp-elastic scattering at 400-880 MeV obtained at Nuclotron, Nucl.Phys.Proc.Suppl. 245 (2013) 271.

[66] Yu.Gurchin et al. The cross-section in dp-elastic scattering at the energies of 500-MeV, 700-MeV and 880-MeV obtained at the internal target station of nuclotron, Phys.Part.Nucl.Lett. 10 (2013) 243.

[67] M.Janek et al., Experimental and simulated dp breakup reaction data at 300, 400 and $500 \mathrm{MeV}$, EPJ Web Conf. 81 (2014) 06005.

[68] S.M.Piyadin et al., Experiment on the study of the $d p \rightarrow$ ppn reaction at 300-500 MeV of the deuteron energy at ITS at Nuclotron, Nucl.Phys.Proc.Suppl. 245 (2013) 177.

[69] K. Suda et al., Development of deuteron polarimeter at Internal Target Station of Nuclotron, AIP Conf.Proc. 915 (2007) 920.

[70] Measurement of analyzing powers for d-p elastic scattering at the Internal Target Station of the Nuclotron, AIP Conf.Proc. 1011 (2008) 241.

[71] T. Uesaka et al., Deuteron beam polarimeter in the nuclotron ring and analyzing power for the d-p elastic scattering at 880-MeV, CNS Report 79 (2009) Tokyo.

[72] Yu.V. Gurchin et al., Detection equipment for investigating d p elastic scattering at internal target of nuclotron in the framework of DSS project, Phys.Part.Nucl.Lett. 8 (2011) 566. 
[73] N.E. Booth et al., Proton-deuteron elastic scattering at 1.0 GeV/c, Phys.Rev. D4 (1971) 1261.

[74] J.C. Alder et al., Elastic p d scattering at 316, 364, 470, and $590 \mathrm{MeV}$ in the backward hemisphere, Phys. Rev. C6 (1972) 2010. 